\title{
Influence of reproductive status on the daily rhythms of oxidative stress markers in Ovis aries
}

Giuseppe Piccione*, Claudia Giannetto, Francesco Fazio, Anna Assenza, Giovanni Caola

Department of Experimental Sciences and Applied Biotechnology, Laboratory of Veterinary Chronophysiology, Faculty of Medical Veterinary, University of Messina, 98168 Messina, Italy

Received 02 December 2009; Accepted 22 January 2010

\begin{abstract}
Changes in circadian rhythms of dROMs, Oxy-ads and SHp during reproductive stages were studied in Comisana ewes. Twelve ewes were divided in two equal groups. The experimental group consisted of ewes undergoing gestation and lactation following artificial insemination and the control group consisted of non-pregnant ewes. Blood samples were collected every $3 \mathrm{~h}$ over a $24 \mathrm{~h}$ period, 20 days before insemination, on days 100 and 140 of pregnancy, on days 10,30 and 200 post-partum and during the dry period. In the control group, blood samples were collected on the same days and with the same procedures as those used for the experimental group. A significant effect of time on all parameters studied was observed in the experimental group. Daily rhythms of the parameters studied were observed in the control group in all experimental conditions, and in the experimental group during pre-pregnancy and dry periods. We conclude that the reproductive status of sheep affects oxidative stress markers in blood and their circadian rhythms.
\end{abstract}

Keywords: Oxidative stress • Daily rhythm • Ewes • Physiological stages

(C) Versita Sp. z 0.0 .

\section{Introduction}

Circadian rhythmicity is an inherent property of living systems and constitutes an essential part of their internal and external temporal order. The appearance of reproducible and stable circadian rhythms of high amplitude, and with a characteristic phasing with respect to other biological processes and the external environment, is believed to guarantee an optimal functioning of the biological system, with maximum efficiency, performance and welfare [1].

Reactive oxygen species (ROS), known also as free radicals, are molecules characterized by the presence of unpaired electrons in their valence orbitals, which make them highly reactive. Through this mechanism, ROS are able to damage numerous cellular molecules, such as proteins, lipids, nucleic acids, amino acids, carbohydrates and vitamins [2]. Physiologically, the free radicals supply the immune system in the defense against bacteria; in case of excessive production that cannot be counteracted by cytoprotective mechanisms, there is an "oxidative stress" that promotes the insurgence of serious pathologies as a result of the degenerative damage of the cellular structures [3-6].

The concept that circadian rhythmicity and redox state are necessarily and intimately linked is widely accepted [7]. Circadian rhythms control several behaviors through neural networks, hormones and gene expression [8]. One of these outputs in invertebrates, vertebrates and plants is the stress resistance behavior. Gene expression that governs this behavior, essential for maintaining a robust anti-oxidative defense, varies daily 
showing circadian rhythmicity $[9,10]$. Because cellular redox state is closely coupled to metabolism [11], this effect of redox state on the circadian pacemaker or on the transcriptional activity of clock proteins indicates that metabolic activity may affect the phase, and perhaps even the functioning, of the circadian clock $[12,13]$. The high-yield production schedules to which livestock is subjected involves physiological phenomena, such as gestation and lactation, that determine endocrine and metabolic modifications with exaggerated anabolic and catabolic process. The peripartum period represents a crucial moment of the reproductive cycle of lactating ruminants, and, as a matter of fact, the metabolic and immunological status of animals changes rapidly and intensively between late pregnancy and early lactation. In previous studies Piccione et al. [14,15] demonstrated a significant effect of lactation stage on oxidative status of dairy cows and ewes, showing that high oxidative processes occur during the milking period in ruminants. A recent study conduced on non pregnant ewes showed circadian rhythms of oxidative stress markers [16]. Given the remarkable zoo-economic impact of metabolic stress, the aim of this study was to bring new knowledge in the chronophysiology of oxidative stress, and to investigate the influence of the different reproductive stages on the circadian rhythm of free radicals and anti-oxidant power in ewes, in order to optimize productive performance and animal welfare.

\section{Experimental Procedures}

For this study, we used twelve pluriparous Comisana ewes that were 3 years old, mean body weight $52.1 \pm 3.2 \mathrm{~kg}$, clinically healthy, free from internal and external parasites, in good nutritional condition (BCS: $2.61 \pm 0.1)$, homogenous for production and estrous synchronized. They were divided in two equal groups. The experimental group consisted of ewes undergoing gestation and lactation following artificial insemination and the control group consisted of non-pregnant ewes. During the trial period, all animals were kept under a natural photoperiod and environmental temperature in a farm located in Sicily $\left(38^{\circ} 7^{\prime} \mathrm{N} ; 13^{\circ} 22^{\prime} \mathrm{E}\right)$ at an altitude of 300 meters above sea level (Table 1). Thermohygrometric recordings were conducted inside the box throughout the entire study by means of a data logger (Gemini, Chichester, West Sussex, UK) placed in the middle of the box at $1 \mathrm{~m}$ height from the floor.

All animals were fed twice a day with straw and hay from a first-crop polyphyte meadow. Water was available ad libitum. The animals of the experimental group were fed twice a day with a mix of cereals formulated according to their physiological and productive stage: pregnancy (crude protein $=17.90 \%$, crude oil $=2.80 \%$, crude fiber $=8.60 \%$, ash $=8.0 \%$ ), lactation (crude protein $=14.30 \%$, crude oil $=3.90 \%$, crude fiber $=7.90 \%$, ash $=8.60 \%$ ), and were milked twice a day by means of a lactating machine. Mean milk production was $1800 \mathrm{~g} /$ day at day 10 of lactation; $2000 \mathrm{~g} /$ day at day 30 of lactation and $900 \mathrm{~g} /$ day at day 200 of lactation.

Blood samples were collected every $3 \mathrm{~h}$ over $24 \mathrm{~h}$ period, starting at 8:00 on day 1 and finishing at 8:00 on day 2, over 7 different reproductive periods: 20 days before insemination (pre pregnancy), day 100 and 140 of pregnancy, day 10, 30 and 200 post-partum and dry period (day 230 post partum). In the control group blood samples were collected on the same days and with the same procedures as for the experimental group (samples 1-7). For each subject, blood was collected from the jugular vein using a vacutainer without anticoagulant. Blood samples were then centrifuged at 1500 r.p.m. for 15 minutes, and the obtained sera were immediately analyzed by means of a Slim spectrophotometer (Seac Florence, Italy), for the assessment of the following parameters: d-ROMs, OXY-Adsorbent and SHp. Free radicals (d-ROMs) and the anti-oxidant power (OXY-Adsorbent and SHp) were assessed with the so-called "spin trap" system, with molecules reacting with free radicals resulting in complexes visible with a spectrophotometer. The d-ROMs test assesses the concentration of hydroperoxides (R-OOH), a class of reactive metabolites of oxygen, in a biological sample (serum, plasma, tissues and cells). The OXY-Adsorbent assesses the anti-oxidant power of the plasmatic barrier by measuring its ability to contrast the oxidative

\begin{tabular}{llccc}
\hline Experimental condition & $\begin{array}{l}\text { Data } \\
\text { (month) }\end{array}$ & $\begin{array}{c}\text { Photoperiod } \\
\text { (sunrise-sunset/h) }\end{array}$ & $\begin{array}{c}\text { Temperature } \\
\left.\text { (min-max/ }{ }^{\circ} \mathrm{C}\right)\end{array}$ & $\begin{array}{c}\text { Humidity } \\
\text { (\%) }\end{array}$ \\
\hline \hline 1/pre-pregnancy & October & $06: 30-17: 00$ & $17-22$ & $65-84$ \\
2/100 days pregnancy & February & $06: 10-18: 10$ & $10-14$ & $71-90$ \\
3/140 days pregnancy & April & $06: 15-19: 50$ & $11-17$ & $76-93$ \\
4/10 days post partum & April & $06: 00-20: 00$ & $14-20$ & $60-85$ \\
5/30 days post partum & May & $05: 30-20: 20$ & $17-25$ & $55-78$ \\
6/200days post partum & November & $06: 00-17: 20$ & $16-18$ & $72-85$ \\
7/dry period & December & $06: 30-17: 00$ & $9-14$ & $60-87$ \\
\hline
\end{tabular}

Table 1. Natural photoperiod, ambient temperature and relative humidity observed during the different experimental conditions. 

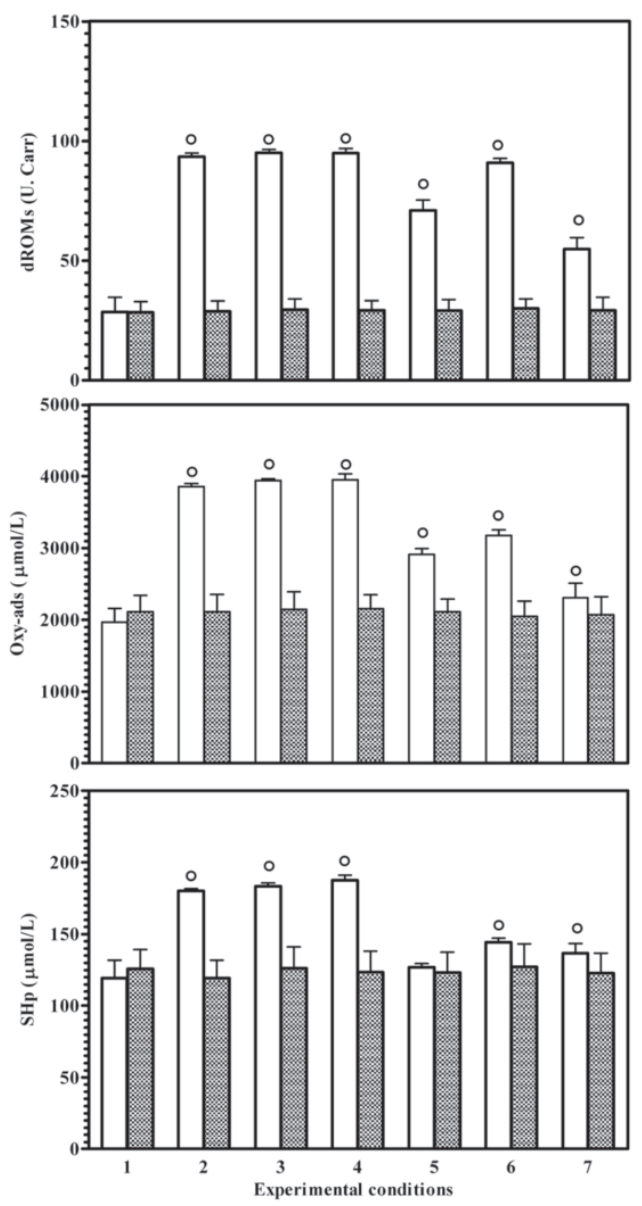

Experimental

Control

Figure 1. Mean of the daily patterns of dROMs, Oxy-ads and SHp observed during the 7 different experimental conditions in the experimental and control groups, with their statistical differences. Significance: ${ }^{\circ}$ vs. Control.

action of hypochlorous acid. This acid is a highly oxidant compound that, in physiological conditions, is synthesized from activated polymorphonucleated leucocytes and acts as an oxidant against bacteria attacks. The SHp test assesses the thiol anti-oxidant plasma barrier, which contrasts the propagation of the perioxydative processes by inactivating both the alkoxyl and the hydroxide radicals.

All the results were expressed as mean $\pm S D$. Twoway ANOVA was used to determine the statistical effect of time of day and reproductive status on the oxidative status markers (dRoms, Oxy-ads, SHp). If ANOVA showed an acceptable level of significance (2alpha=0.05), Bonferroni's test was applied for post hoc comparison. One-way ANOVA was applied on the mean daily values to compare the 7 experimental conditions. The data were analyzed using the software STATISTICA 7 (StatSoft Inc.). In addition, a trigonometric statistical model was applied to the average values of each time series, so as to describe the periodic phenomenon analytically, by characterizing the main rhythmic parameters according to the single cosinor procedure [17]. Four rhythmic parameters were determined: mean level, amplitude (the difference between the peak, or trough, and the mean value of a wave), acrophase (the time at which the peak of a rhythm occurs), and robustness (strength of rhythmicity). For each parameter, the mean level of each rhythm was computed as the arithmetic mean of all values in the data set (9 data points), the amplitude of a rhythm was calculated as half the range of oscillation, which in turn was computed as the difference between the peak and trough. Rhythm robustness was computed as a percentage of the maximal score attained by the chi-square periodogram statistic for ideal data sets of comparable size and $24 \mathrm{~h}$ periodicity [18]. Robustness greater than $55 \%$ is above noise level and indicates statistically significant rhythmicity.

\section{Results}

The application of a two-way ANOVA showed a significant effect of reproductive status between the experimental and control groups (Figure 1) and a significant effect of time of day on the oxidative markers. In particular, in the experimental group a significant effect of time of day was observed on dRoms, Oxy-ads and SHp during the pre-pregnancy and dry periods. In the control group a significant effect of time of day was observed in all parameters for the 7 experimental conditions (Figure 2).

The application of a one-way ANOVA on the mean daily values observed during the 7 different reproductive periods showed a significant effect of reproductive status on the mean values of the circulation concentration of oxidative stress markers in the experimental group. In the control group the application of a one-way ANOVA on the mean daily values observed during the 7 different experimental conditions no showed a significant effect of the experimental conditions on the mean values of the circulation concentration of oxidative stress markers (Table 2).

In the experimental group, during pre pregnancy and dry periods, the application of the periodic model and the statistical analysis of the cosinor procedure throughout the time series studied showed a robust daily rhythm of the parameters studied. The acrophases of the daily 


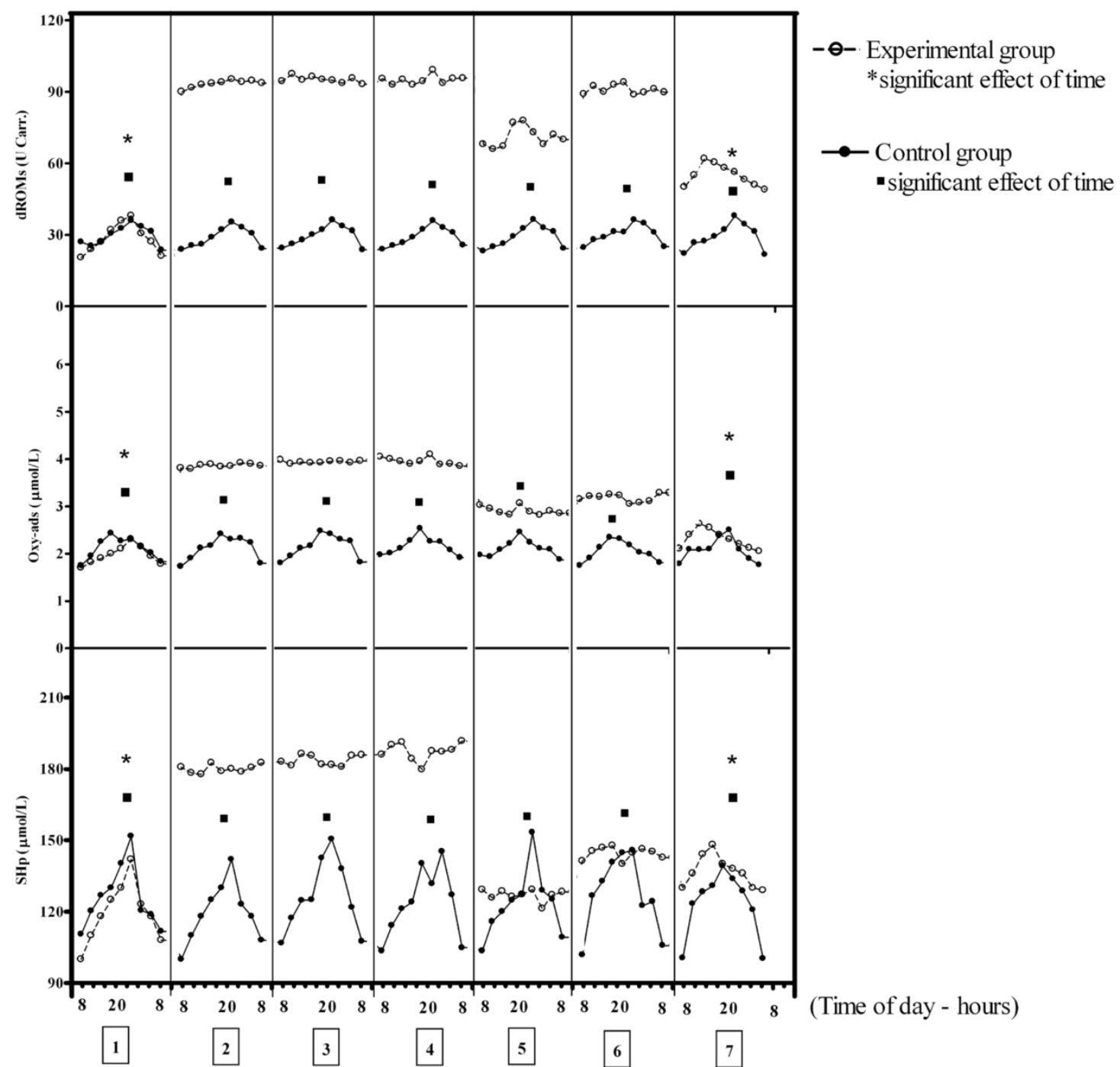

Experimental conditions

Legend: 1:Pre-pregancy; 2:100 days of pregnancy; 3:140 days of pregnancy;

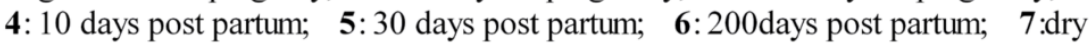

Figure 2. Daily pattern of oxidative stress markers during the different experimental conditions in the experimental and control groups.

\begin{tabular}{|c|c|c|c|c|c|c|c|c|}
\hline Group & Parameter & $\begin{array}{c}1 / \\
\text { pre-pregnancy }\end{array}$ & $\begin{array}{l}2 / \text { day } 100 \text { of } \\
\text { pregnancy }\end{array}$ & $\begin{array}{l}3 / \text { day } 140 \text { of } \\
\text { pregnancy }\end{array}$ & $\begin{array}{c}\text { 4/day } 10 \text { post } \\
\text { partum }\end{array}$ & $\begin{array}{c}\text { 5/day } 30 \text { post } \\
\text { partum }\end{array}$ & $\begin{array}{c}\text { 6/day } 200 \text { post } \\
\text { partum }\end{array}$ & $\begin{array}{l}7 / \\
\text { dry }\end{array}$ \\
\hline \multirow{3}{*}{ Experimental } & dROMs (U. Carr) & $28.49 \pm 6.16$ & $93.33 \pm 1.62^{*}$ & $95.9 \pm 1.31^{*}$ & $95.04 \pm 1.89^{\star}$ & $71.02 \pm 4.31^{* \bullet \bullet}$ & $90.88 \pm 1.86^{*}$ & $55.01 \pm 4.60^{\circ}$ \\
\hline & Oxy-ads $(\mu \mathrm{mol} / \mathrm{L})$ & $1967.00 \pm 192.30$ & $3859.00 \pm 41.55^{*}$ & $3939.00 \pm 25.45^{*}$ & $3954.00 \pm 82.23^{*}$ & $2911.00 \pm 87.70^{\circ}$ & $3174.00 \pm 81.87^{\circ}$ & $2306.00 \pm 204.9^{\circ}$ \\
\hline & $\mathrm{SHp}(\mu \mathrm{mol} / \mathrm{L})$ & $119.30 \pm 12.58$ & $180.00 \pm 1.72^{\star}$ & $183.50 \pm 2.22^{*}$ & $187.30 \pm 3.73^{*}$ & $127.00 \pm 2.43^{\circ \bullet \bullet}$ & $144.50 \pm 2.59^{\circ}$ & $136.80 \pm 6.55^{\circ}$ \\
\hline \multirow{3}{*}{ Control } & dROMs (U. Carr) & $29.28 \pm 4.57$ & $28.89 \pm 4.19$ & $29.57 \pm 4.28$ & $29.25 \pm 4.09$ & $29.08 \pm 4.61$ & $30.15 \pm 3.96$ & $29.25 \pm 5.42$ \\
\hline & Oxy-ads $(\mu \mathrm{mol} / \mathrm{L})$ & $2107.00 \pm 233.10$ & $2109.00 \pm 246.20$ & $2146.00 \pm 248.20$ & $2155.00 \pm 193.20$ & $2107.00 \pm 179.10$ & $2050.00 \pm 210.00$ & $2072.00 \pm 247.20$ \\
\hline & SHp $(\mu \mathrm{mol} / \mathrm{L})$ & $125.60 \pm 13.39$ & $119.30 \pm 12.58$ & $126.00 \pm 15.13$ & $123.50 \pm 14.47$ & $123.10 \pm 14.20$ & $127.20 \pm 15.80$ & $122.90 \pm 13.81$ \\
\hline
\end{tabular}

Table 2. Mean values $( \pm \mathrm{SD}$ ) of oxidative stress markers studied in the experimental and control groups during experimental conditions, with their statistical differences.

Significance: *versus condition 1/pre-pregnacy

'versus condition 2/day 100 pregnancy

-versus condition 3/day 140 pregnancy

-versus condition 4/day 10 post partum

versus all other experimental conditions 
rhythm of oxidative stress markers were observed during the evening and afternoon hours, for the pre-pregnancy and dry period, respectively. In the control group the periodic pattern of the parameter studied was observed in all sample periods (Figure 3 ).

a)

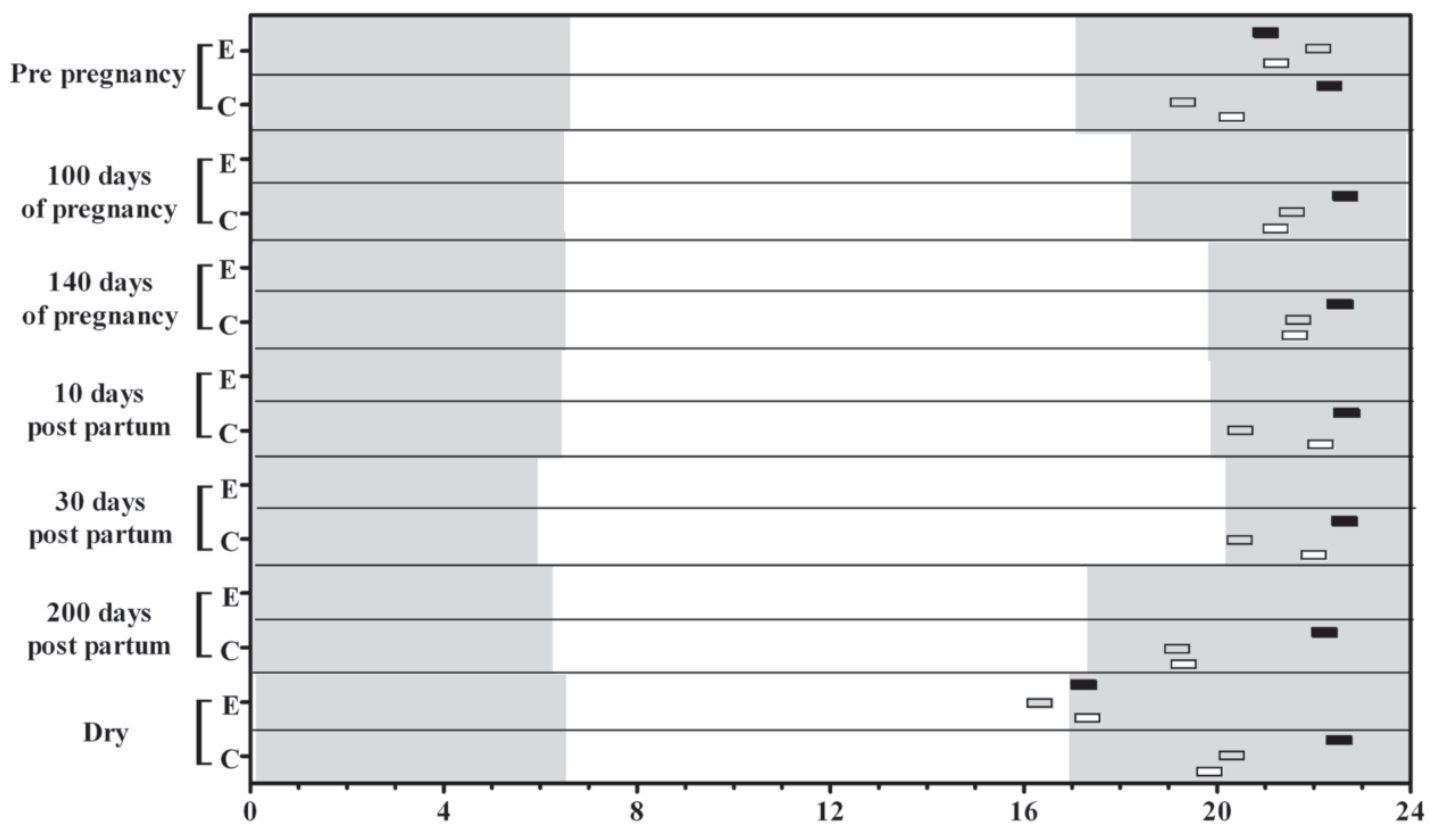

b)

Time of day (hours)

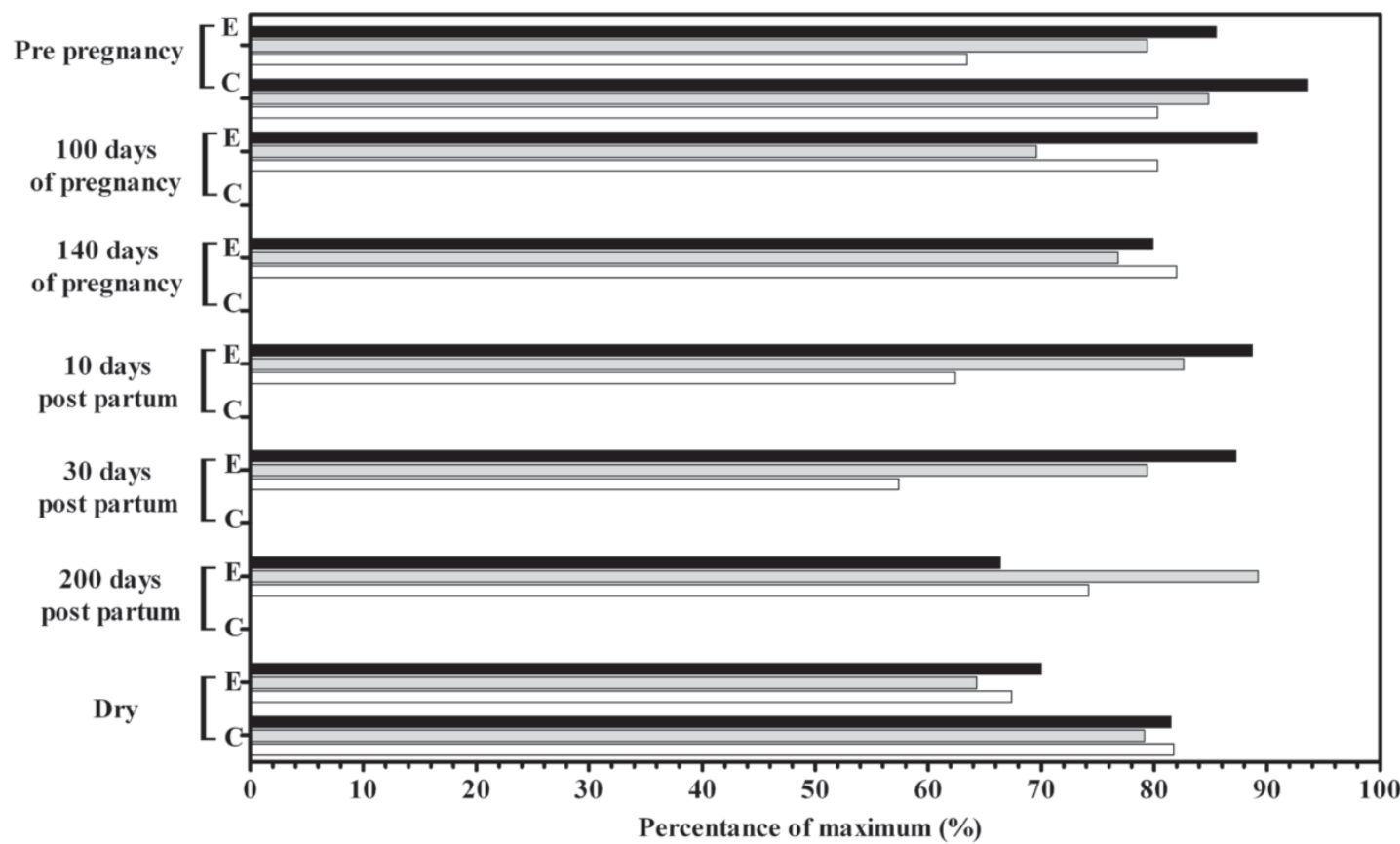

Figure 3. Acrophases (a) and robustness (b) of circadian rhythms of dRoms ( $\mathbf{m})$, Oxy-ads ( $\square$ ) and SHp ( $\square$ ) observed in the experimental (E) and control (C) groups during the different experimental conditions 


\section{Discussion}

Our results showed the occurrence of daily rhythms of oxidative stress markers in ewes, with nocturnal acrophase. These rhythms were lost during the reproductive period, and in particular on the days which demonstrate high circulating concentrations of oxidative markers.

As previously observed in cows and ewes [14,15], a significant effect of gestation and milking on the oxidative stress markers was observed. Pregnancy is a physiological state accompanied by a high metabolic demand and an increased requirement for tissue oxygen [19]. This increased oxygen requirement, observed during pregnancy, rose the rate of ROS production [20], that was accompanied by the increase of Oxy-ads and $\mathrm{SH}$ p serum levels. These increases were present also during the first 10 days of lactation. Parturition itself may induce the increase in ROS production due to changes in oxygen use and pressure. The rate of milk yield with stage of lactation is strongly influenced by the rate of cell death by apoptosis in the lactating gland [21].

The response of organisms to the stress induced by negative energetic balance secondary to lactation favors lipomobilization and gluconeogenesis, which, in terms of oxidative balance, means free radical excess. In milking animals, oxidative stress physiologically acts at the mammary gland level as a cellular turnover modulator, and high levels of oxidative stress might be involved in the control of milk yield [21]. An imbalance between the formation and detoxification of oxygen radicals may occur during early lactation, which is known to be associated with an increase in oxidative metabolism to provide metabolites for the production of milk [22].

Our results confirm the high energetic requirements of the milking period in ewes. The same trend of oxidative stress markers during the gestation and lactation periods testifies to the compensative response of the organism to oxidative stress. Also, our results showed robust daily rhythms of dRoms, Oxy-ads and

\section{References}

[1] Weinert D., Waterhouse J., The circadian rhythm of core temperature: Effects of physical activity and aging, Physiol. Behav., 2007, 90, 246-256

[2] Czesnikiewicz-Guzik M., Konturek S.J., Loster B., Wisniewska G., Majewski, S., Melatonin and its role in oxidative stress related diseases of oral cavity, $\mathrm{J}$. Physiol. Pharmacol., 2007, 58, 5-19

[3] Mccord J.M., The evolution of free radicals and oxidative stress, Am. J. Med., 2000, 108, 652-657
$\mathrm{SHp}$ in non pregnant ewes. These rhythms persisted in all experimental conditions in the control group, while they were lost during gestation and lactation, appearing again during the dry period, in the experimental group. This could be due to an influence of reproductive hormones, with oestradiol and progesterone acting to preserve mammary ductal and alveolar integrity during the dry period, while allowing a degree of apoptosis and cell replacement [21].

In a previous study conduced on experimental animals, a link was demonstrated between oxidative stress and circadian rhythms. In particular, it was found that the circadian clock is sensitive to oxidative stress [12]. We can suppose that the oxidative stress can influence the circadian clock in ewes, even though further studies are necessary to investigate the influence of ROS and anti-oxidative power on the suprachiasmatic nuclei or the peripheral oscillator.

This is the first study reporting the rhythmicity of oxidative markers in ewes and their susceptibility to endogenous stress. This suggests that the physiological response to oxidative stress is regulated by the circadian clock in ewes, and that the response to endogenous stress alters this response. Previous studies have reported a rhythmic susceptibility of oxidative stress to exogenous stressors such as hydrogen peroxide, xenobiotics or pathogens in flies [9,23-25].

In conclusion, dRoms production in sheep occurs in a circadian manner, and with nocturnal acrophase. Peak anti-oxidative action precedes the dRoms acrophase by about 2 hours. This underlines the important role in effective defense of organism and in maintaining the balance between dRoms production and removal. The level of production in ewes might affect the oxidative status of the organism, and the high activity in the tissue associated with gestation and lactation might mask the basal rhythms in oxidative stress processes.

[4] Freidovich I., Fundamental aspects of reactive oxygen species, or what's the matter with oxygen?, Ann. NY Acad. Sci., 1999, 893, 13-20

[5] Matsuo M., Kaneko T, The chemistry of reactive oxygen species and related free radicals, In: Radak Z., (Ed), Free radicals in exercise and aging, Human kinetics, Leeds: Pudsey, United Kindom, 2000

[6] Saleh M.A., Al-Salahy M.B., Sanousi S.A., Corpuscular oxidative stress in desert sheep naturally deficient in copper, Small Rum. Res., 2008, 80, 33-38 
[7] Foyer C.H., The redox state and circadian rhythms, Ann. Bot., 2002, 89, 500-501

[8] Berger J., A two-clock model of circadian timing in the immune system of mammals, Pathol. Biol., 2008, 56, 286-291

[9] Krishnan N., Davis A.J., Giebultowicz J.M., Circadian regulation of response to oxidative stress in Drosophila melangaster, Biochem. Biophys. Res. Commun., 2008, 374, 299-303

[10] Simonetta S.H., Romanowski A., Minniti A.N., In Estrosa N.C., Golombek D.A., Circadian stress tolerance in adult Caenorhabditis elegans, J. Comp. Physiol. A, Neuroethol. Sens. Neural. Behav. Physiol., 2008, 194, 821-828

[11] Harper M.E., Bevilacqua L., Hagopian K., Weindruch R., Ramsey J.J., Ageing, oxidative stress, and mitochondrial uncoupling, Acta Physiol. Scand., 2004, 182, 321-331

[12] Zheng X., Yang Z., Yue Z., Avarez J.D., Sehgal A., FOXO and insulin signaling regulate sensitivity of the circadian clock to oxidative stress, Proc. Natl. Acad. Sci. USA, 2007, 104, 15899-15904

[13] Mongrain V., Cermakian N., Clock genes in health and diseases, J. Appl. Biomed., 2009, 7, 15-33

[14] Piccione G., Borruso M., Fazio F., Grasso F., Caola G., Oxidative stress evaluation during milking period in the ewes, J. Appl. Anim. Res., 2006, 29, 109-112

[15] Piccione G., Borruso M., Giannetto C., Morgante M., Giudice E., Assessment of oxidative stress in dry and lactating cows, Acta Agric. Scand. A, 2007, 57, 101-104

[16] Piccione G., Giannetto C., Fazio F., Pennisi P., Caola G., Evaluation of total locomotor activite and oxidative markers daily rhythms in sheep, Biol. Rhythm Res., 2010, DOI: 10.1080/09291010903408225

[17] Nelson K., Tong J.L., Lee J.K., Halberg F., Methods for cosinor rhythmometry, Chronobiologia, 1979, 6, 305-323
[18] Refinetti R., Non-stationary time series and the robustness of circadian rhythms, J. Theor. Biol., 2004, 227, 571-581

[19] Spätling L., Fallenstein F., Huch A., Huch R., Rooth G., The variability of cardiopulmonary adaptation to pregnancy at rest and during exercise, Br. J. Obstet. Gynaecol., 1992, 99, 1-40

[20] Halliwell B., Gutteridge J.M., The antioxidants of human extracellular fluids, Arch. Biochem. Biophys., 1990, 280, 1-8

[21] Stefanon B., Colitti M., Gabai G., Knight C.H., Wilde C.J., Mammary apoptosis and lactation persistency in dairy animals, J. Dairy Res., 2002, 69, 37-52

[22] Löhrke K., Viergutz T., Kanitz W., Göllnitz K., Hurtienne A., Schweigert F.J., High milk yield in dairy cows associated with oxidant stress, J. Vet. Res., 2004, 8, 70-78

[23] Gorbacheva V.J., Kondratov R., Zhang S., Cherukuri S., Gudkov A.V., Takahashi J.S., et al., Circadian sensitivity to the chemotherapeutic agent cyclophosphamide depends on the functional status of the CLOCK/BMAL1 transactivation complex, Proc. Natl. Acad. Sci. USA, 2005, 102, 3407-3412

[24] Gachon F., Olela F.F., Schaad O., Descombes P., Schibler U., The circadian PAR-domain basis leucin zipper transcription factors DBP, TEF, and HLF modulate basal and inducible xenobiotic detoxification, Cell Metab., 2006, 4, 25-36

[25] Lee J.E., Edery I., Circadian regulation in the ability of Drosophila to combat phatogenic infections, Curr. Biol., 2008, 18, 195-199 